\title{
USE OF COGNITIVE STRATEGIES IN RATS: THE ROLE OF ESTRADIOL AND ITS INTERACTION WITH DOPAMINE.
}

\author{
Matthew G. Quinlan, Dema Hussain, and Wayne G. Brake \\ Center for Studies in Behavioral Neurobiology (CSBN) \\ Department of Psychology \\ Concordia University \\ Montreal, QC, Canada
}

Address Correspondence to:

Wayne Brake, Ph.D.

Center for Studies in Behavioral Neurobiology

Concordia University

SP-244, 7141 Sherbrooke Street West

Montreal, QC, Canada, H4B 1R6

E-mail: wayne.brake@concordia.ca

Phone: (514) 848-2424 ext. 5451

FAX: (514) 848- 2817 


\begin{abstract}
Accumulating evidence suggests a role for estrogen in the use of a particular cognitive strategy when solving a maze task. In order to confirm the role of estrogen in this phenomenon, ovariectomized (OVX) female rats receiving either high $(\sim 90 \mathrm{pg} / \mathrm{ml})$ or low $(\sim$ $32 \mathrm{pg} / \mathrm{ml}$ ) circulating levels of $17 \beta$-estradiol benzoate (E2) performed a plus maze task for a reward. Consistent with previous research, OVX rats receiving low levels of E2 utilized a striatum-mediated response strategy while OVX rats administered high levels of E2 employed a hippocampus-mediated place strategy. Furthermore, following a systemic injection of a moderate dose of either a dopamine D1 (SKF 83566, $0.1 \mathrm{mg} / \mathrm{kg}$ IP) or D2 (raclopride, $0.5 \mathrm{mg} / \mathrm{kg} \mathrm{IP}$ ) receptor antagonist, low E2 rats were seen to use the opposite strategy and exercise a hippocampus-mediated place strategy in order to obtain the reward. At the same doses, high E2 rats did not change from using a place strategy. At a lower dose, these drugs shifted high E2 rats such that they showed an equal propensity for either strategy; this was not observed in low E2 rats. These results corroborate previous findings that E2 plays a significant role in the use of either a response or place strategy when solving a maze for a reward. In addition, the shift in strategy after dopamine receptor blockade implies the importance of central dopamine function in selecting a cognitive strategy to solve such tasks. It is suggested that estrogen alters cognitive strategy not only by improving hippocampal function, but also by altering dopamine-regulated striatal function.
\end{abstract}

Key Terms: Cognitive strategy, response learning, memory, SKF 83566, raclopride, plus maze 


\section{Introduction}

There is extensive evidence demonstrating the effects of estrogen $(\mathrm{E})$ on cognitive performance (for reviews see Hampson, 1995; Sherwin, 2003). These effects have been confirmed in varied learning and memory paradigms and across several species. For instance, a review of these benefits in a clinical population (e.g. Sherwin, 2003) reports that high levels of naturally circulating ovarian hormones enhance performance in female-typical cognitive tasks such as verbal memory and fine-motor skills. In addition, E replacement therapy in menopausal and hysterectomized women maintains or re-invigorates the same cognitive abilities. Although the reported effects of $\mathrm{E}$ on performance in a cognitive task are well established, E may also have additional effects including an influence on the utilization of a particular strategy necessary to solve a task than solely the level of performance.

In fact, recent evidence in rodents points to an effect of $E$ on the relative efficacy of employing a hippocampus-mediated 'place' strategy versus a striatum-mediated 'response' strategy when solving a cognitive task (Korol and Kolo, 2002; Korol, Malin, Borden, Busby, and Couper-Leo, 2004). Evidence for multiple learning and memory systems primarily based in these brain areas comes from numerous studies.

It was originally demonstrated in male rats that selective inactivation of the hippocampus affects the expression of a place strategy while damage to the caudate nucleus results in deficient response learning in a cross-maze task (Packard and McGaugh, 1996) and in the Morris water maze (McDonald and White, 1994). White and McDonald (2002) suggest that interference with hippocampal processing impaired the ability of rats to learn the location of a food reward in an 8-arm radial maze during a win-shift task but had no effect on learning in a win-stay task (White and McDonald, 2002). Whereas lesions of the dorsal 
striatum attenuated performance in the win-stay, but not the win-shift, condition. In an 8-arm radial maze, retrieval of a reward in win-shift tasks is primarily dependent on the development of a spatial map of the maze and its immediate environment, or a place strategy. Conversely, a win-stay task requires the formation of a stimulus-response relationship without the involvement of any extra-maze cues, or a response strategy (White and McDonald, 2002). Moreover, spatial working memory is impaired in rats with hippocampal damage but not striatal damage (Kesner, 1990). However, response memory of a directional turn is attenuated in rats with striatal lesions but not hippocampal lesions (Cook and Kesner, 1988). During response tasks in which striatal lesions significantly impair performance, rats with damaged hippocampi, but intact striatal function, exhibit an enhanced performance compared to normal rats (Matthews, Simson, and Best, 1995). Studies such as these suggest a dissociation, yet potential interaction, of the neural bases for particular cognitive strategies. In addition, the effectiveness of a particular strategy in solving a task is enhanced if relevant information from the alternative structure is made unavailable. Investigations of neurotransmitter release have also provided evidence for multiple memory systems. McIntyre et al. (2003) showed that acetylcholine release during acquisition of a dual solution T-maze task is increased in both the hippocampus and striatum of rats. However, this release was greater in the hippocampus of those rats employing a place strategy whereas in rats utilizing a response strategy striatal acetylcholine release was greater.

Importantly, evidence indicates that these distinct learning and memory systems are differentially regulated by the presence of E. An early study by Williams and colleagues (1990) established that neonatal hormonal manipulations alter the strategy by which rats solve a task in adulthood. In addition, OVX rats receiving E replacement acquired a place 
task more quickly than those receiving the vehicle which, in turn, performed better in a response task (Korol and Kolo, 2002). The removal of a static cue signaling the location of a platform in the Morris water maze interfered with the ability of animals receiving vehicle to complete the task but did not affect $\mathrm{E}$ treated animals (Daniel and Lee, 2004). Most convincingly, Korol et al. (2004) have shown that when naturally cycling rats in a plus maze are free to use a place or response strategy with which to solve the task, rats in the proestrus phase (high E) were biased towards utilization of a place strategy whereas rats in the estrus phase (low E) were more likely to employ a response strategy. A large body of research has been conducted examining the E-mediated enhancement of hippocampal function and its effects on cognitive performance and strategy solution. Yet, little research has investigated the role of striatal dopamine (DA) and its interaction with differential internal hormonal states during the selection of a cognitive strategy.

There is converging evidence implicating striatal DA in response-based learning. Electrophysiological studies indicate that circuits within the basal ganglia promote the acquisition of a task through trial and error learning in which a particular behavioral response is shaped by reward-related contingencies (Graybiel, 2005). Moreover the striatum contains response- and reward-related neural representations (Mizumori, Yeshenko, Gill, and Davis, 2004). Chemical and structural lesions to the dorsal striatum interfere with response learning tasks (Compton, 2004; McDonald and White, 1994; Packard and McGaugh, 1996) and similar damage impaired acquisition of plus maze and T-maze tasks in cue-deficient environments (Chang and Gold, 2004). Anatomical evidence shows that the striatum contains the highest density of DA D1 receptors (D1R) and DA D2 receptors (D2R) in the brain, both within the caudate-putamen and the nucleus accumbens (Boyson, McGonigle, and Molinoff, 
1986). A recent study by Daniel et al. (2006) showed that E interacts with D2R but not D1R to affect performance in a response learning task.

Although such results support a role for striatal DA in the performance of responsebased cognitive tasks, there is a lack of evidence examining estrogenic influence on DA in the selection of a cognitive strategy by which these tasks are solved. In order to investigate this interaction, here rats were trained in a dual solution $\mathrm{T}$ maze task that allows for the utilization of either a place or response strategy. Adult female rats were ovariectomized and administered levels of either low or high 17ß-estradiol (E2). Based on findings by Korol et al. (2004) using the same task, it was expected that during a no-drug probe trial most low E2 rats would solve the maze using a response strategy and most high E2 rats would employ a place strategy. Furthermore, in order to determine the influence of E2 on DAergic mediation of strategy selection, both groups of animals received systemic injections of either a D1R or $\mathrm{D} 2 \mathrm{R}$ antagonist prior to subsequent probe trials. Considering that performance in a response learning task is reportedly sensitive to D2R but not D1R antagonism (Daniel et al., 2006), both were examined here to determine whether the same pattern is seen in use of strategy.

These effects were investigated with a hormone administration regimen that was intended to mimic the levels of E during the estrous cycle. That is, the low E2 group received constant levels of E2 similar to that seen during the estrus phase and the high E2 group received constant low E2 plus daily injections resulting in peaks of E2 similar to that seen during the proestrus phase. 
Materials and Methods

\section{Subjects and Surgery:}

Subjects included 30 female Sprague-Dawley rats (Charles River, St. Constant, QC, Canada), approximately three months of age. All rats weighed between 225-250 grams. Before training began, animals were housed in pairs in polyurethane shoebox cages and maintained on a reverse 12h:12h light/dark cycle with lights off from 0900-2100. Standard lab chow and water were available ad libitum until training began. The rats were handled daily from time of arrival until completion of the experiment.

Approximately one week after arrival, all rats were anesthetized using a mixture of ketamine $(50 \mathrm{mg} / \mathrm{ml})$ and xylazine $(4 \mathrm{mg} / \mathrm{ml})$ in a $4: 3$ ratio $(1 \mathrm{ml} / \mathrm{kg}$, IP) and bilaterally ovariectomized using a standard aseptic procedure through a dorsal incision. Post-surgical care included administration of the antibiotic Baytril (0.03 ml/animal, SC), the analgesic banamine (0.03 ml/animal, SC), and 0.9\% saline ( $3 \mathrm{ml} / \mathrm{animal}, \mathrm{SC})$. Subsequent to surgery, all animals were allowed to recover in their home cages for several days until surgery for implantation of Silastic tubes. All animal protocols were in accordance with guidelines established by the Canadian Council on Animal Care and were approved by the Concordia University Animal Research Ethics Committee.

\section{Hormone administration and measurement:}

Animals were randomly assigned to one of two groups; low E2 ( $\mathrm{n}=15)$ or high E2 $(n=15)$. Three days after the ovariectomy surgeries, all animals were anesthetized using 
Halothane gas (4\% for induction and $2 \%$ for maintenance) and a Silastic tube (1cm long; i.d. $1.47 \mathrm{~mm}$; o.d. $1.96 \mathrm{~mm}$ ) containing 5\% 17ß-estradiol benzoate (Sigma Chemical Co., St Louis, MO) in cholesterol (Sigma) was subcutaneously implanted in the nape of the neck. This has been reported to produce a serum concentration of approximately $20 \mathrm{pg} / \mathrm{ml}$ consistent with naturally circulating low levels of E such as those seen during the estrus phase of the rat estrous cycle (Mannino, South, Inturrisi, and Quinones-Jenab, 2005). For the high E2 group, in addition to the subcutaneous implants, daily subcutaneous injections were given of $17 \beta$-estradiol benzoate $(10 \mu \mathrm{g} / \mathrm{kg})$ dissolved in sesame oil (Sigma) designed to achieve levels seen during the proestrus phase of the estrous cycle $(75-90 \mathrm{pg} / \mathrm{ml})$. During the same period all animals in the low E2 group received daily subcutaneous injections of sesame oil as a control $(1 \mathrm{ml} / \mathrm{kg})$. All injections began two days before habituation training and occurred between 1200-1400h each day.

Approximately 30 days following the implantation of the Silastic tubes, i.e. at the completion of behavioral testing, blood was collected from the tail vein at $1200 \mathrm{~h}$. Blood samples were immediately centrifuged and plasma was collected and stored at $-20{ }^{\circ} \mathrm{C}$ until assayed. E2 was measured using a commercially available ELISA kit (Immuno-Biological Laboratories Inc., Minneapolis, MI). The assay antibodies have 100\% cross-reactivity with E2 and $0.2 \%$ and $0.05 \%$ cross-reactivity with estrone and estriol respectively. The range of the assay is between $0-2000 \mathrm{pg} / \mathrm{ml}$ and the reported inter-assay variation is $7-9 \%$.

\section{DA antagonist administration:}

Animals received systemic injections of DA antagonists on separate days. The selective D1R antagonist SKF 83566 (Sigma) was administered in a moderate dose of 0.1 
$\mathrm{mg} / \mathrm{kg}$ and a lower dose of $0.01 \mathrm{mg} / \mathrm{kg}$. Raclopride, a selective D2R antagonist, was administered in a moderate dose of $0.5 \mathrm{mg} / \mathrm{kg}$ and lower dose of $0.1 \mathrm{mg} / \mathrm{kg}$. These doses were selected because they are in the mid and low ranges for modifying behaviors in attention and learning tasks for both SKF 83566 (Domenger and Schwarting, 2006; Salamone, Arizzi, Sandoval, Cervone, and Aberman, 2002; Serafim and Felicio, 2001) and raclopride (Shaham and Stewart, 1996; Wise and Carlezon, 1994). Both drugs were dissolved in $0.9 \%$ saline at room temperature and stored no longer than four days at $4{ }^{\circ} \mathrm{C}$. All drugs were administered intraperitoneally (IP) 30 minutes prior to the onset of behavioral testing and animals were allowed a washout period for a minimum of $24 \mathrm{~h}$ between each drug probe trial.

Apparatus, Modified Plus Maze:

All training was carried out in a polyurethane plus maze placed on a table one meter above the floor. The maze was constructed with black walls extending $23 \mathrm{~cm}$ above a wire grid floor that is $10.5 \mathrm{~cm}$ wide and enclosed with removable clear polyurethane roof panels. The start and probe arms do not have roof panels on them. The modified plus maze had four arms arranged at $90^{\circ}$ angles around a $14 \times 14 \mathrm{~cm}$ central chamber; two goal arms, a training start arm, and a probe start arm, all of which were $75 \mathrm{~cm}$ in length (figure 1). Entrance from the central chamber to all arms could be occluded by black polyurethane guillotine gates which could be lifted by the experimenter using a string from a remote location.

Throughout the training trials the probe start arm was blocked off from the central chamber creating a T-shaped maze (figure 1A). At the commencement of the probe trial, the probe arm was unblocked and the original start arm was blocked creating an alternative T- 
shaped maze exactly $180^{\circ}$ in orientation (figure 1B). Each start arm contained a start-box $30 \mathrm{~cm}$ in length which blocked access to the central chamber by a black polyurethane guillotine gate halfway down the arm. Each goal arm contained a white ceramic bowl in which a food reward (Kellogg's Froot Loops ${ }^{\circledR}$ ) could be placed. Froot Loop crumbs were placed underneath both goal arms of the maze during all trials to prevent any confounds due to odor cues. Extra-maze cues were very obvious and included a large dark poster on a plain white wall opposite wall to wall blue metal cupboards on the other wall. The experimenter in a white lab coat stood in the same position during all trials. For all trials, the maze was kept stationary in a position relative to all extra-maze cues throughout testing. All testing took place under dim red light illumination.

\section{Procedure:}

Habituation. Approximately five days after ovariectomy surgery all animals were placed on food restriction which lasted until completion of the experiment and maintained body weight at $90 \%$ of free-feeding weight. All training was performed at the beginning of the dark phase of the light/dark cycle commencing at 09:00. Beginning seven days after implantation of the Silastic tubes, all animals were given three days of habituation to the modified plus maze with the probe trial start arm blocked off creating a T-shaped maze (see figure 1A). Habituation consisted of 15-min sessions in the maze with Froot Loops scattered throughout the apparatus.

Training. Following habituation, all rats began acquisition training. Each animal was assigned to receive a food reward, half of a Froot Loop, in either the right or left goal arm. The baited goal arm was counterbalanced across rats such that half the rats in each group 
received the reward in the right arm and half in the left arm. For each particular rat the Froot Loop was always in the same goal arm. Training consisted of 10 daily trials per rat beginning in the start box of the training arm. Rats were placed in the start-box behind the guillotine gate and were allowed to rear and look and sniff around the room while waiting to start.. Once the gate panels of both goal arms were raised the animal was released from the startbox and allowed a free choice to enter either the right or left goal arm. A trial was ended when all four limbs of a rat crossed into a goal arm and the gate could be closed, or when a 2min time limit was reached. Rats that chose correctly were allowed to eat the food reward in the arm before being returned to their home cage. Rats that chose incorrectly were allowed to investigate the empty food bowl before being taken out of the arm and placed into the home cage. Inter-trial intervals were 10-30 sec during which time another rat completed its trial. Rats were considered to have reached criterion after performing $8 / 10$ correct trials for three days in a row.

Probe testing. On the third day of criterion performance the rat was given a probe trial starting from the probe arm (figure 1B). The rat was placed in the probe start arm, $180^{\circ}$ opposite the blocked training start arm, and the experimenter returned to the standard location. From this position, the goal arm gates were lifted and the animal was released and allowed to enter either the right or left arm. If an animal made the same directional turn in its choice of an arm for which it had been rewarded in the training phase, then it was scored as a response strategy. If an animal made an opposite directional turn in its choice of an arm towards the same spatial location of its reward during the training phase, then it was scored as a place strategy. 
$D 1 R$ and D2R Antagonism. Each rat was injected with a particular type and dose of drug in a counterbalanced manner. All drug trials were given subsequent to achievement of criterion and completion of the initial probe trial. One day after the no-drug probe trial each rat was injected with a drug and trained again for 10 trials then given the drug probe trial. No subsequent drug injections were given for at least $24 \mathrm{~h}$. Every rat maintained its performance of $8 / 10$ correct trials during all drug trials.

\section{Statistical Analyses:}

This was a mixed design with E2 level as a between subjects factor and drug as a within subjects factor. Because the dependent variable was percent of rats using a type of cognitive strategy, probe and drug trial data were analyzed using non-parametric statistics. A Chi-square $\left(\chi^{2}\right)$ analysis was conducted to compare high and low E rats in their use of strategy in the no-drug probe trial. Multiple $\chi^{2}$ tests were also conducted for these two groups for each of the drug probe trials at both doses of SKF 83566 and raclopride to test whether there was a significant probability of using one strategy over another in each individual case. To test whether there was a significant difference in strategy use in high and low E2 groups, a Kruskal-Wallis test was conducted. In order to test the effects of DA antagonists, McNemar tests were conducted using a binomial for small frequencies to compare no-drug probe trials with each of the respective doses of SKF 83566 and raclopride.

To control for the effects, if any, of repeated testing in this task, a Cochrane Q test was conducted on percent of rats using either strategy across all 5 days of probe and drug trials. Finally, to determine if there were any differences in learning this task, a two-tailed ttest was conducted on the number of days to reach criterion for low and high E2 groups. 


\section{Results}

Plasma E2 levels, task acquisition and no-drug probe test:

The mean ( \pm S.E.M.) plasma E2 level for the low E2 rats was $32.62 \mathrm{pg} / \mathrm{ml}( \pm 7.05)$ and for the high E2 rats it was $90.44 \mathrm{pg} / \mathrm{ml}( \pm 19.70)$. These levels are within range for estrus and proestrus respectively.

By the time the first criterion day was reached the entire testing session for each rat lasted no more than 15 min and trials were completed in $20 \mathrm{sec}$ or less, but most often within 5 sec. There were no significant differences $(t=-0.259, \mathrm{p}=0.79)$ between the high and low E2 groups in the number of days to reach criterion. The means $( \pm$ S.E.M) were $4.68( \pm 0.32)$ for Low E2 and 4.81 ( \pm 0.37) for High E2. In addition, there was no effect of trial test day on prevalence of using either a place or a response strategy $(Q=4.857, \mathrm{p}=0.302)$.

Figure 2 illustrates that on the no-drug probe trial, high E2 rats were significantly more likely to employ a place strategy $\left(80 \%, \chi^{2}=0.02\right)$ than a response strategy $(20 \%)$. In contrast, Low E2 rats showed a non-significant but strong trend to utilize a response strategy $\left(73 \%, \chi^{2}=0.071\right)$ over a place strategy $(27 \%)$. A Kruskal-Wallis test revealed a significant difference between high and low E2 groups in the strategy used $(\mathrm{p}=0.004)$.

\section{DIR antagonist probe trials (SKF 83566):}

As can be seen in figure 3 (top) D1R blockade by a $0.1 \mathrm{mg} / \mathrm{kg}$ dose of SKF 83566 in the low E2 group resulted in a significant shift $(\mathrm{McNemar}, \mathrm{p}=0.039)$ in the method used to solve the probe trial from a majority of rats using a response strategy in the no drug probe to using a place strategy $\left(80 \%, \chi^{2}=0.02\right)$. Conversely, low dose administration of $0.01 \mathrm{mg} / \mathrm{kg}$ 
SKF 83566 had no effect in comparison to the no drug probe trial as there was a significant probability that these rats would employ a response strategy $\left(87 \%, \chi^{2}=0.005\right)$ over a place strategy.

As can be seen in figure 4 (top), after systemic administration of a $0.1 \mathrm{mg} / \mathrm{kg}$ dose of the selective D1R antagonist SKF 83566, high E2 animals maintained use of their no-drug strategy and were significantly more likely to employ a place strategy $\left(80 \%, \chi^{2}=0.02\right)$ than a response strategy (20\%). However, administration of a $0.01 \mathrm{mg} / \mathrm{kg}$ dose resulted in these animals being equally likely to use either a place strategy (40\%) or a response strategy (60\%) in order to solve the task.

\section{D2R antagonist probe trials (Raclopride):}

Figure 3 (bottom) illustrates that comparable to intermediate D1R antagonism, systemic administration of $0.5 \mathrm{mg} / \mathrm{kg}$ raclopride in low $\mathrm{E} 2$ animals resulted in a significant shift of cognitive strategy $(\mathrm{McNemar}, \mathrm{p}=0.008)$ as significantly fewer animals exercised a response strategy $\left(13 \%, \chi^{2}=0.005\right)$ compared to a place strategy $(87 \%)$. In contrast to the effect of a low dose D1R antagonism in low E2 animals, low dose $(0.1 \mathrm{mg} / \mathrm{kg}) \mathrm{D} 2 \mathrm{R}$ antagonism with raclopride resulted in a significant shift of cognitive strategy (McNemar, $\mathrm{p}=$ $0.021)$ as significantly more low E2 animals utilized a place strategy $\left(80 \%, \chi^{2}=0.02\right)$ over a response strategy $(20 \%)$.

As can be seen in figure 4 (bottom), systemic injection of a $0.5 \mathrm{mg} / \mathrm{kg}$ dose of the D2R antagonist raclopride did not effect of strategy selection in high E2 animals as the majority employed a place strategy $\left(80 \%, \chi^{2}=0.005\right)$ over a response strategy $(20 \%)$ which 
is how these rats responded in the no drug condition. After administration of $0.1 \mathrm{mg} / \mathrm{kg}$ of raclopride, however, rats were equally likely to utilize either a place strategy (40\%) or a response strategy (60\%), similar to the effects subsequent to low dose D1R antagonism.

\section{Discussion}

The present results confirm previous findings (Korol et al., 2004) that demonstrate variations in the levels of E2 in female rats strongly influence the probable use of a particular cognitive strategy when solving a maze for a reward. The data show that chronic low levels of E2 will bias an animal towards the utilization of a striatum-mediated 'response' strategy and repeated high levels of E2 will bias a rat towards use of a hippocampus-mediated 'place' strategy.

The most dramatic effects of DA antagonism in the current study were observed in the low E2 rats. Both doses of raclopride and the higher dose of SKF 83566 caused the low E2 rats to switch from a majority employing a response strategy in the no-drug trial, to most rats employing a place strategy. The only exception was in response to the lower dose of the D1R antagonist, SKF 83566, which did not change the strategy used by most low E2 rats.

The effects of DA antagonism on high E2 rats were the same for both the D1R antagonist, SKF 83566, and the D2R antagonist, raclopride. Neither of these drugs had any effect on strategy selection at the higher doses. That is, high E2 rats maintained a greater probability of using a place strategy when administered the higher doses of these drugs. On the other hand, high E2 rats responded to the lower doses of both drugs by being equally likely to use a place or a response strategy, showing no probability of using one strategy over 
another. It has been shown that high levels of $\mathrm{E}$ when acutely administered will increase DA release in the striatum (Becker, 1990) and E2 will increase both D1R and D2R binding (Di Paolo, Poyet, and Labrie, 1981; Levesque and Di Paolo, 1989).

Strategy use was strongly influenced by systemic administration of either a D1R or D2R antagonist and these effects were different depending on whether rats were administered high or low E2. Although it has been shown that E2 increases binding of both D1R and D2R in the striatum (Di Paolo et al., 1981; Levesque and Di Paolo, 1989), a recent behavioral study found that E2 interacted with D2R but not D1R antagonism. In examining the effects of $\mathrm{E} 2$ on performance in a response task, D2R antagonism disrupted performance to a greater extent in the high E2 OVX rats than in no E2 OVX controls. Conversely, there was no difference in high $\mathrm{E} 2$ or no $\mathrm{E} 2$ in rats in response to D1R antagonism (Daniel et al., 2006). This is not in agreement with the results reported here insofar as antagonism of both receptor subtypes had an effect on strategy selection. On the other hand, in the study by Daniel and colleagues (2006) the measured outcome was performance in a response task, whereas here the measure was the use of either a place or a response strategy to solve a task that could be completed using either strategy.

These results suggest that a hippocampus-dependent place strategy is more likely to be used by rats receiving high E2. The strategy use of High E2 rats was not altered following administration of a moderate dose of either a D1R or D2R antagonist. In addition, Low E2 rats administered either a moderate dose of D1R or both doses of D2R antagonist were biased to use a place strategy. Numerous studies demonstrate a beneficial influence of high $\mathrm{E}$ on hippocampal function and hippocampus-dependent behaviors across several species (e.g. McEwen, Akama, Alves, Brake, Bulloch, Lee, Li, Yuen, and Milner, 2001). For instance, 
higher levels of acute E enhance hippocampal dendritic spine density (Woolley and McEwen, 1992) and synaptic proteins in rats (Brake, Alves, Dunlop, Lee, Bulloch, Allen, Greengard, and McEwen, 2001) as well as in rhesus macaques (Choi, Romeo, Brake, Bethea, Rosenwaks, and McEwen, 2003). The potential influence of acute $\mathrm{E}$ on learning and memory has also been demonstrated insofar as it facilitates long term potentiation (Cordoba Montoya and Carrer, 1997; Warren, Humphreys, Juraska, and Greenough, 1995) and attenuates long term depression (Day and Good, 2005) in the hippocampus. These studies suggest that $\mathrm{E}$ increases hippocampal function, the data presented here suggest that the bias toward hippocampus-dependent strategy is not only influenced by E alone but also by its interaction with central DA function.

Low E2 rats show a bias toward use of a striatum-dependent response strategy. In addition, following administration of a low dose D1R antagonist, low E2 rats were more likely to maintain use of a response strategy. Although less is known concerning estrogenic effects on striatum-based learning, evidence suggests that high E levels impair learning and performance in tasks dependent on striatal function. For example, high E2 administration to OVX female rats impaired acquisition of tasks dependent upon response learning such as a response variant of the T-maze (Davis, Jacobson, Aliakbari, and Mizumori, 2005) and a cuebased Morris water maze subsequent to removal of the cue (Daniel and Lee, 2004). E2 infusion into the striatum also impaired a cue-deficient version of a Y-maze (Zurkovsky, Brown, Boyd, Fell, and Korol, 2007) . Moreover, low levels of E2 have been shown to improve learning and performance in tasks that depend on striatal functioning such as nonspatial working memory in an alternating T-maze task (Wide, Hanratty, Ting, and Galea, 2004) and in a radial arm maze (Holmes, Wide, and Galea, 2002). 
Studies in this lab using gonadally-intact female rats have shown that during proestrus, a period of high E, performance in a latent inhibition task is interrupted (Quinlan, Graffe, Duncan, and Brake, 2006) and high E replacement in OVX rats disrupts latent inhibition (Nofrey, Ben-Shahar, and Brake, 2007). Lubow (1997) has reviewed evidence strongly suggesting latent inhibition is a task dependent on striatal DA function.

Additionally, the effects of E on pre-pulse inhibition (PPI), another striatal DA-dependent task, are evident in both clinical and experimental populations. Investigations of PPI in human subjects indicate that sensorimotor gating performance is reduced during the luteal phase of the menstrual cycle when circulating levels of E are high (Jovanovic, Szilagyi, Chakravorty, Fiallos, Lewison, Parwani, Schwartz, Gonzenbach, Rotrosen, and Duncan, 2004). Studies in rats also show a significant reduction of PPI during high E proestrus females as compared to males or to females in the diestrus or estrus phases of the estrous cycle when E levels are low (Koch, 1998).

There is also physiological data to support the idea that E may affect striatal function. For example, E is known to affect DA function in the striatum (for review see, Becker, 1999) and recent evidence suggests that E2 inhibits gamma- aminobutyric acid (GABA) release in the striatum (Hu, Watson, Kennedy, and Becker, 2006).

In summary, the present results are a confirmation of previous findings demonstrating the important influence of differential E2 levels in the use of a strategy during performance of a cognitive task. In addition, a comparably relevant role of DA in this process is implicated by the shift in strategy selection subsequent to modulation of D1R and D2R. However, this is not merely an isolated effect of hormonal influence or neurotransmitter activity but rather an interaction between these two systems that significantly impacts the 
behavior of a rat in a learning and memory task. Dependent on the levels of E2 in the brain, there are differential modulations of the DAergic activity which significantly alter the approach by which a rat will solve a task for a reward.

\section{Acknowledgements:}

This work was supported by the Natural Sciences and Engineering Research Council of Canada and the Canada Foundation for Innovation (W.G.B.). The authors thank Barbara Woodside and Jane Stewart for their valuable comments on an earlier version of this manuscript. The assistance of Nafissa Ismail with the ovariectomy surgeries is greatly appreciated. 
Figure Captions

Figure 1: The modified plus maze used in this experiment as adapted from Korol et al. (2004). A) Rats were initially trained to receive a reward in either the left or right goal arm. Upon reaching criterion, rats were then placed in the opposite arm B) for the start of the probe trial.
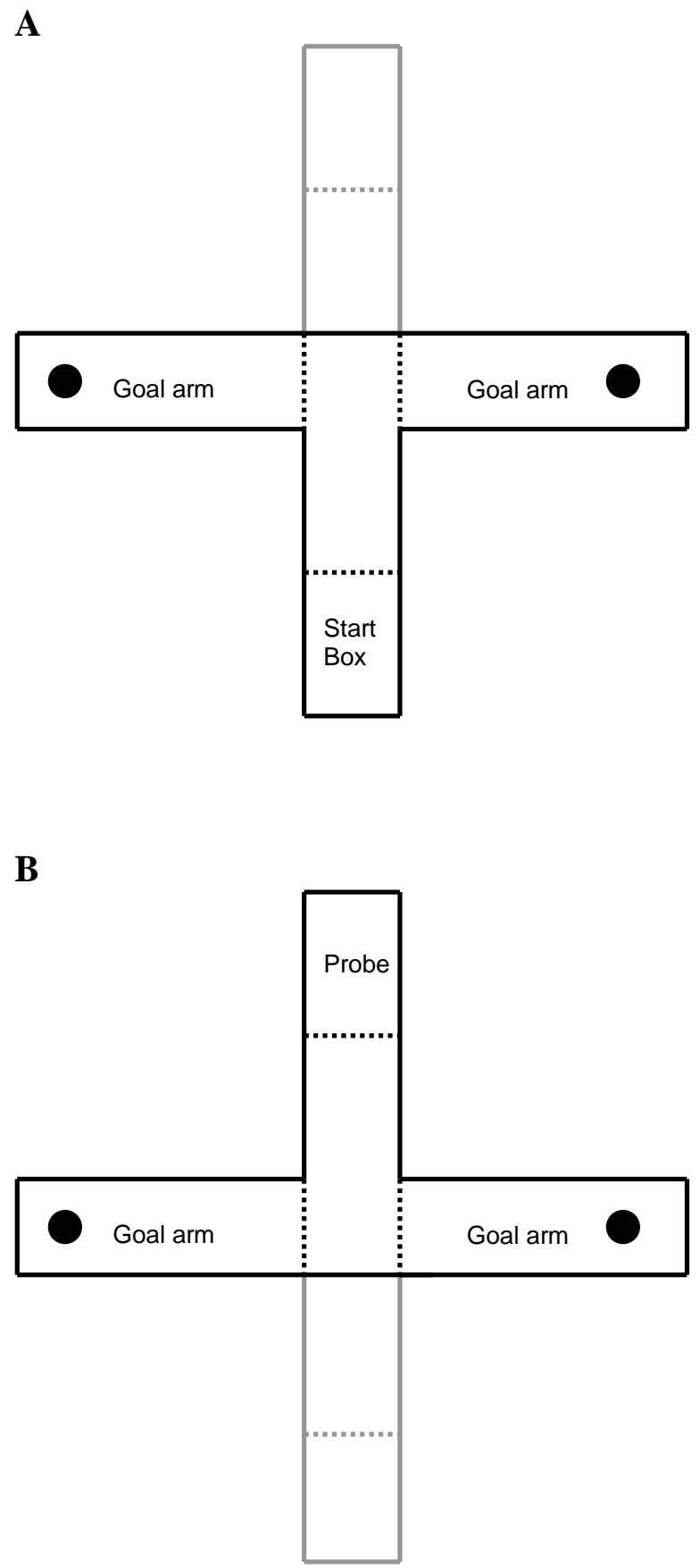
Figure 2: Strategy use in ovariectomized rats administered either high or low estradiol. A significantly greater proportion of High estradiol rats employed a place strategy $*\left(\chi^{2}=0.02\right.$, Chi-Square test) over a response strategy. High E2 rats significantly differed from Low E2 rats in strategy use ${ }^{\#}(p=0.004$, Kruskal-Wallis test $)$.

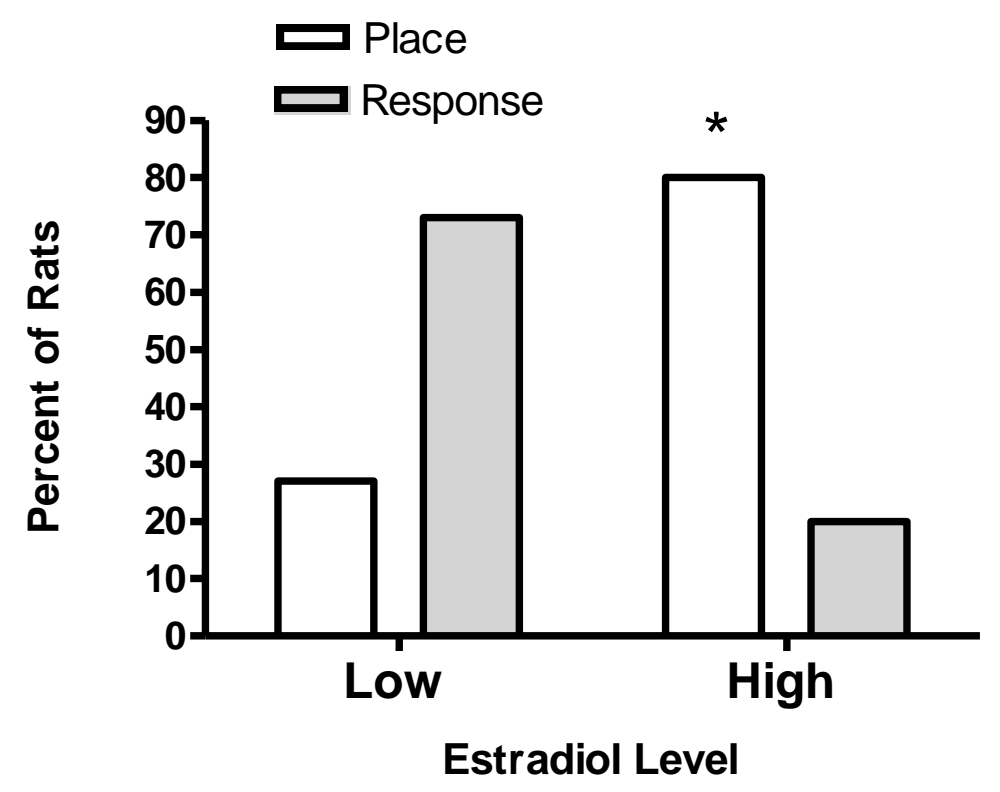


Figure 3: Strategy use in ovariectomized rats administered low estradiol during the no-drug probe trial and in response to doses of a dopamine $\mathrm{D}_{1}$ receptor antagonist (SKF 83566) and a dopamine $\mathrm{D}_{2}$ receptor antagonist (raclopride). * significant $(\mathrm{p}<0.05)$ difference in percent of rats using response strategy versus place strategy (Chi Square). \# significant switch in strategy compared to probe trial (McNemar).

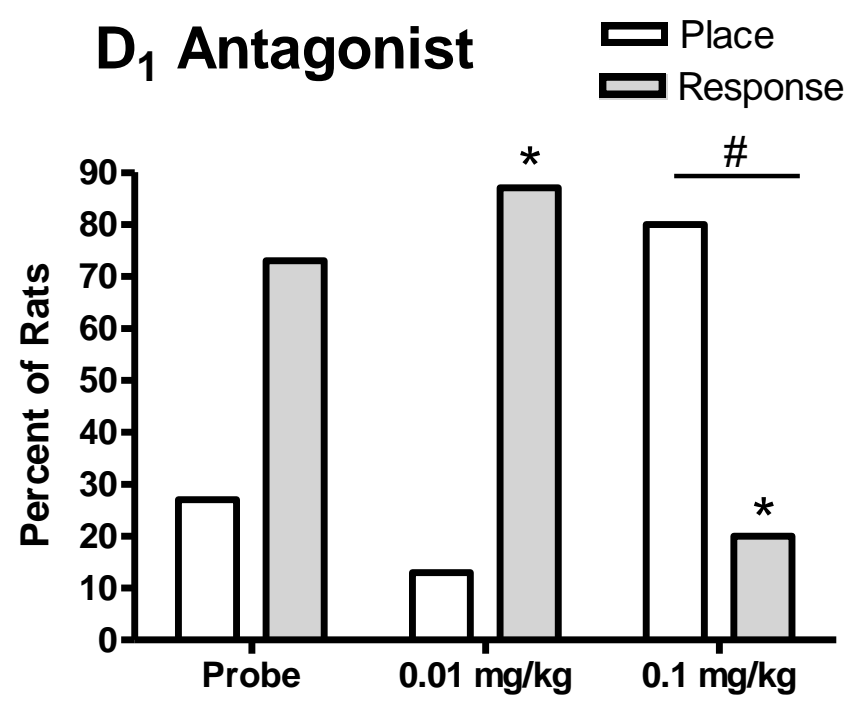

\section{$D_{2}$ Antagonist}

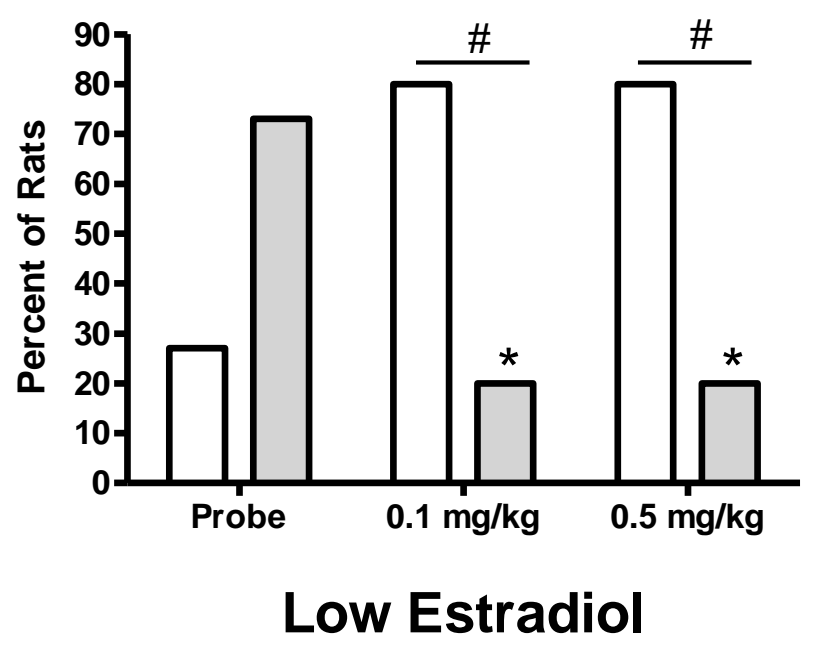


Figure 4: Strategy use in ovariectomized rats administered high estradiol during the no-drug probe trial and in response to doses of a dopamine $\mathrm{D}_{1}$ receptor antagonist (SKF 83566) and a dopamine $\mathrm{D}_{2}$ receptor antagonist (raclopride). * significant $(\mathrm{p}<0.05)$ difference in percent of rats using response strategy versus place strategy (Chi Square).

\section{$D_{1}$ Antagonist $\begin{aligned} & \text { Place } \\ & \text { Response }\end{aligned}$}

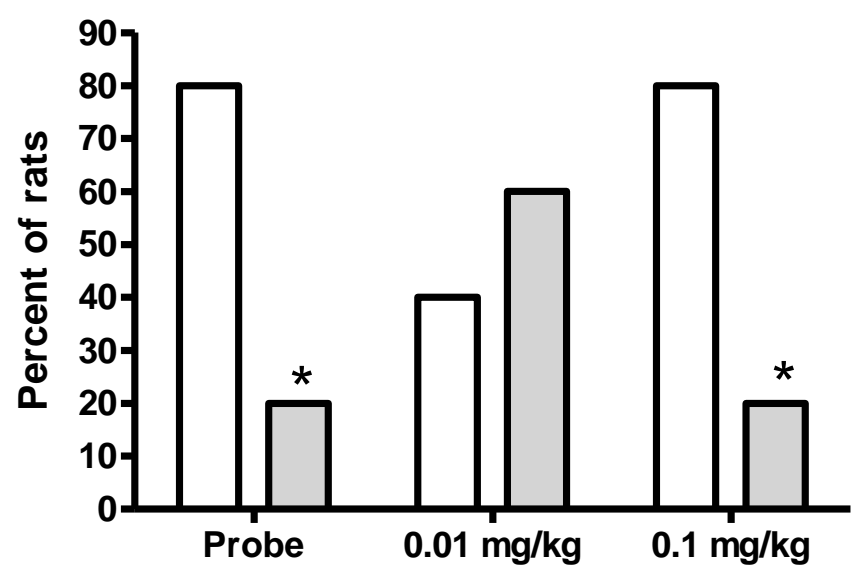

\section{$D_{2}$ Antagonist}

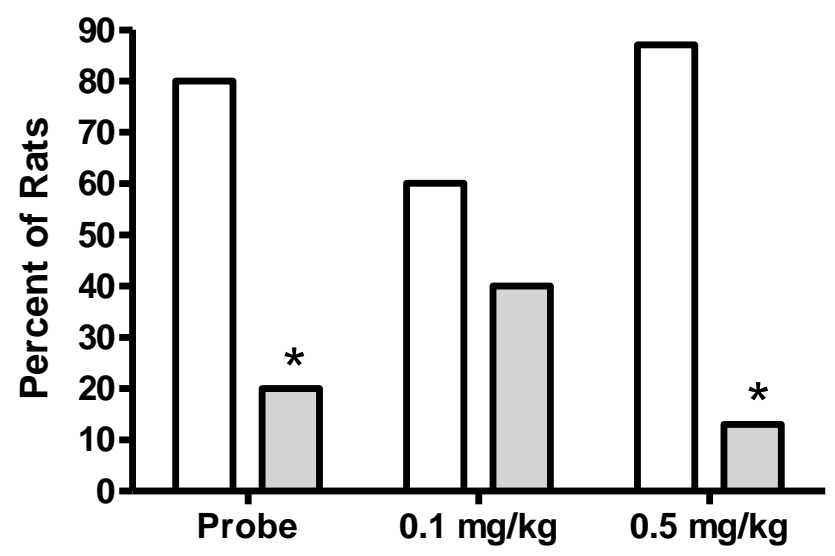

High Estradiol 


\section{References}

Becker, J. B. (1990). Estrogen rapidly potentiates amphetamine-induced striatal dopamine release and rotational behavior during microdialysis. Neurosci Lett 118(2), 169-71.

Becker, J. B. (1999). Gender differences in dopaminergic function in striatum and nucleus accumbens. Pharmacol Biochem Behav 64(4), 803-12.

Boyson, S. J., McGonigle, P., and Molinoff, P. B. (1986). Quantitative autoradiographic localization of the D1 and D2 subtypes of dopamine receptors in rat brain. J Neurosci 6(11), 3177-88.

Brake, W. G., Alves, S. E., Dunlop, J. C., Lee, S. J., Bulloch, K., Allen, P. B., Greengard, P., and McEwen, B. S. (2001). Novel target sites for estrogen action in the dorsal hippocampus: an examination of synaptic proteins. Endocrinology 142(3), 1284-9.

Chang, Q., and Gold, P. E. (2004). Inactivation of dorsolateral striatum impairs acquisition of response learning in cue-deficient, but not cue-available, conditions. Behav Neurosci 118(2), 383-8.

Choi, J. M., Romeo, R. D., Brake, W. G., Bethea, C. L., Rosenwaks, Z., and McEwen, B. S. (2003). Estradiol increases pre- and post-synaptic proteins in the CA1 region of the hippocampus in female rhesus macaques (Macaca mulatta). Endocrinology 144(11), 4734-8.

Compton, D. M. (2004). Behavior strategy learning in rat: effects of lesions of the dorsal striatum or dorsal hippocampus. Behav Processes 67(3), 335-42.

Cook, D., and Kesner, R. P. (1988). Caudate nucleus and memory for egocentric localization. Behav Neural Biol 49(3), 332-43.

Cordoba Montoya, D. A., and Carrer, H. F. (1997). Estrogen facilitates induction of long term potentiation in the hippocampus of awake rats. Brain Res 778(2), 430-8.

Daniel, J. M., and Lee, C. D. (2004). Estrogen replacement in ovariectomized rats affects strategy selection in the Morris water maze. Neurobiol Learn Mem 82(2), 142-9.

Daniel, J. M., Sulzer, J. K., and Hulst, J. L. (2006). Estrogen increases the sensitivity of ovariectomized rats to the disruptive effects produced by antagonism of D2 but not D1 dopamine receptors during performance of a response learning task. Horm Behav 49(1), 38-44.

Davis, D. M., Jacobson, T. K., Aliakbari, S., and Mizumori, S. J. (2005). Differential effects of estrogen on hippocampal- and striatal-dependent learning. Neurobiol Learn Mem 84(2), 132-7.

Day, M., and Good, M. (2005). Ovariectomy-induced disruption of long-term synaptic depression in the hippocampal CA1 region in vivo is attenuated with chronic estrogen replacement. Neurobiol Learn Mem 83(1), 13-21.

Di Paolo, T., Poyet, P., and Labrie, F. (1981). Effect of chronic estradiol and haloperidol treatment on striatal dopamine receptors. Eur J Pharmacol 73(1), 105-6.

Domenger, D., and Schwarting, R. K. (2006). The serial reaction time task in the rat: effects of D1 and D2 dopamine-receptor antagonists. Behav Brain Res 175(2), 212-22.

Graybiel, A. M. (2005). The basal ganglia: learning new tricks and loving it. Curr Opin Neurobiol 15(6), 638-44.

Hampson, E. (1995). Spatial cognition in humans: possible modulation by androgens and estrogens. J Psychiatry Neurosci 20(5), 397-404. 
Holmes, M. M., Wide, J. K., and Galea, L. A. (2002). Low levels of estradiol facilitate, whereas high levels of estradiol impair, working memory performance on the radial arm maze. Behav Neurosci 116(5), 928-34.

Hu, M., Watson, C. J., Kennedy, R. T., and Becker, J. B. (2006). Estradiol attenuates the K+induced increase in extracellular GABA in rat striatum. Synapse 59(2), 122-4.

Jovanovic, T., Szilagyi, S., Chakravorty, S., Fiallos, A. M., Lewison, B. J., Parwani, A., Schwartz, M. P., Gonzenbach, S., Rotrosen, J. P., and Duncan, E. J. (2004). Menstrual cycle phase effects on prepulse inhibition of acoustic startle. Psychophysiology 41(3), 401-6.

Kesner, R. P. (1990). Memory for frequency in rats: role of the hippocampus and medial prefrontal cortex. Behav Neural Biol 53(3), 402-10.

Koch, M. (1998). Sensorimotor gating changes across the estrous cycle in female rats. Physiol Behav 64(5), 625-8.

Korol, D. L., and Kolo, L. L. (2002). Estrogen-induced changes in place and response learning in young adult female rats. Behav Neurosci 116(3), 411-20.

Korol, D. L., Malin, E. L., Borden, K. A., Busby, R. A., and Couper-Leo, J. (2004). Shifts in preferred learning strategy across the estrous cycle in female rats. Horm Behav 45(5), 330-8.

Levesque, D., and Di Paolo, T. (1989). Chronic estradiol treatment increases ovariectomized rat striatal D-1 dopamine receptors. Life Sci 45(19), 1813-20.

Lubow, R. E. (1997). Latent inhibition as a measure of learned inattention: some problems and solutions. Behav Brain Res 88(1), 75-83.

Mannino, C. A., South, S. M., Inturrisi, C. E., and Quinones-Jenab, V. (2005). Pharmacokinetics and effects of 17beta-estradiol and progesterone implants in ovariectomized rats. J Pain 6(12), 809-16.

Matthews, D. B., Simson, P. E., and Best, P. J. (1995). Acute ethanol impairs spatial memory but not stimulus/response memory in the rat. Alcohol Clin Exp Res 19(4), 902-9.

McDonald, R. J., and White, N. M. (1994). Parallel information processing in the water maze: evidence for independent memory systems involving dorsal striatum and hippocampus. Behav Neural Biol 61(3), 260-70.

McEwen, B., Akama, K., Alves, S., Brake, W. G., Bulloch, K., Lee, S., Li, C., Yuen, G., and Milner, T. A. (2001). Tracking the estrogen receptor in neurons: implications for estrogen-induced synapse formation. Proc Natl Acad Sci U S A 98(13), 7093-100.

McIntyre, C. K., Marriott, L. K., and Gold, P. E. (2003). Patterns of brain acetylcholine release predict individual differences in preferred learning strategies in rats. Neurobiol Learn Mem 79(2), 177-83.

Mizumori, S. J., Yeshenko, O., Gill, K. M., and Davis, D. M. (2004). Parallel processing across neural systems: implications for a multiple memory system hypothesis. Neurobiol Learn Mem 82(3), 278-98.

Nofrey, B. S., Ben-Shahar, O. M., and Brake, W. G. (2007). Estrogen abolishes latent inhibition in ovariectomized female rats. Brain Cogn in press.

Packard, M. G., and McGaugh, J. L. (1996). Inactivation of hippocampus or caudate nucleus with lidocaine differentially affects expression of place and response learning. Neurobiol Learn Mem 65(1), 65-72. 
Quinlan, M. G., Graffe, N., Duncan, A., and Brake, W. G. (2006). Postpubertal but not prepubertal rats show sex differences in latent inhibition, Society for Neuroscience Abstracts.

Salamone, J. D., Arizzi, M. N., Sandoval, M. D., Cervone, K. M., and Aberman, J. E. (2002). Dopamine antagonists alter response allocation but do not suppress appetite for food in rats: contrast between the effects of SKF 83566, raclopride, and fenfluramine on a concurrent choice task. Psychopharmacology (Berl) 160(4), 371-80.

Serafim, A. P., and Felicio, L. F. (2001). Dopaminergic modulation of grooming behavior in virgin and pregnant rats. Braz J Med Biol Res 34(11), 1465-70.

Shaham, Y., and Stewart, J. (1996). Effects of opioid and dopamine receptor antagonists on relapse induced by stress and re-exposure to heroin in rats. Psychopharmacology (Berl) 125(4), 385-91.

Sherwin, B. B. (2003). Estrogen and cognitive functioning in women. Endocr Rev 24(2), 133-51.

Warren, S. G., Humphreys, A. G., Juraska, J. M., and Greenough, W. T. (1995). LTP varies across the estrous cycle: enhanced synaptic plasticity in proestrus rats. Brain Res 703(1-2), 26-30.

White, N. M., and McDonald, R. J. (2002). Multiple parallel memory systems in the brain of the rat. Neurobiol Learn Mem 77(2), 125-84.

Wide, J. K., Hanratty, K., Ting, J., and Galea, L. A. (2004). High level estradiol impairs and low level estradiol facilitates non-spatial working memory. Behav Brain Res 155(1), 45-53.

Williams, C. L., Barnett, A. M., and Meck, W. H. (1990). Organizational effects of early gonadal secretions on sexual differentiation in spatial memory. Behav Neurosci 104(1), 84-97.

Wise, R. A., and Carlezon, W. A., Jr. (1994). Attenuation of the locomotor-sensitizing effects of the D2 dopamine agonist bromocriptine by either the D1 antagonist SCH 23390 or the D2 antagonist raclopride. Synapse 17(3), 155-9.

Woolley, C. S., and McEwen, B. S. (1992). Estradiol mediates fluctuation in hippocampal synapse density during the estrous cycle in the adult rat. J Neurosci 12(7), 2549-54.

Zurkovsky, L., Brown, S. L., Boyd, S. E., Fell, J. A., and Korol, D. L. (2007). Estrogen modulates learning in female rats by acting directly at distinct memory systems. Neuroscience 144(1), 26-37. 